\title{
CRIAÇÃO DE INOVAÇÃO LOCAL POR SUBSIDIÁRIAS ESTRANGEIRAS A PARTIR DE VANTAGENS ESPECÍFICAS DO AMBIENTE SUBNACIONAL
}

\author{
Cristina Doritta Rodrigues ${ }^{\Omega 1}$, Felipe Mendes Borinif ${ }^{f}$, Muhammad Mustafa Raziq ${ }^{\ddagger}$ e \\ Manuel Portugal Ferreira ${ }^{€}$ \\ $\Omega$ Escola Superior de Propaganda e Marketing - ESPM, São Paulo, (Brasil) \\ £ Universidade de São Paulo - USP, São Paulo, (Brasil) \\ ¥ National University of Sciences and Technology, Islamabad (Pakistan) \\ € Universidade Federal de Lavras - UFLA, Minas Gerais (Brasil)
}

\section{DETALHES DO ARTIGO}

\section{Histórico do Artigo:}

Recebido em: 30 de março de 2020

Aceito: 17 de outubro de 2020

Disponível online: 01 de janeiro de 2021

Sistema de revisão "Double blind review"

Editor Científico

Ilan Avrichir

\section{Palavras-chaves:}

Subsidiárias estrangeiras

Ambiente subnacional

Inovação local

Regressão linear múltipla

\section{RESUMO}

Objetivo - O estudo objetiva examinar os efeitos dos fatores do ambiente subnacional brasileiro - a eficiência do ambiente competitivo subnacional e o embeddedness relacional em cada localidade subnacional - na criação de inovação local em subsidiárias estrangeiras.

Método - A abordagem é quantitativa e a pesquisa baseia-se em dados primários coletados por meio de uma survey com 152 subsidiárias estrangeiras localizadas em treze Estados do Brasil e em dados secundários do World Bank Enterprise Surveys. Os dados são analisados por regressão linear múltipla.

Principais resultados - Os resultados mostram que existe uma associação positiva e significante entre o ambiente competitivo subnacional e inovação local das subsidiárias. Entretanto, não é observada uma associação significativa entre o embeddedness externo subnacional e a inovação local. Além disso, os resultados mostram que as empresas que entram por meio de aquisições estão mais associadas à inovação local.

Relevância/Originalidade - O estudo procura complementar o debate sobre o efeito do ambiente subnacional na inovação local das subsidiárias tendo como contexto o Brasil. A análise em nível subnacional considera a heterogeneidade institucional subnacional e revela variações que poderiam ser desconsideradas por uma análise de nível nacional.

Contribuições teóricas/metodológicas: Embora existam indícios do impacto do ambiente institucional na estratégia e inovação, este estudo no âmbito brasileiro avança em dois pontos. Primeiro, os estudos existentes analisam mercados desenvolvidos e mercados emergentes, porém esses últimos de forma pontual e numa realidade asiática, que apresenta uma considerável diferença do mercado latino americano e brasileiro, destacando-se as peculiaridades do ambiente subnacional brasileiro. Segundo, contribui para os estudos de Geografia Econômica reforçando que cada ambiente subnacional tem suas particularidades, conforme demonstrado na natureza das questões subnacionais no Brasil.

Contribuições sociais / para a gestão: Em termos gerenciais, o estudo chama a atenção para a importância do conhecimento da dinâmica competitiva subnacional quanto à participação da economia informal e estende implicações para políticas públicas. 


\section{INTRODUÇÃO}

A literatura em gestão internacional ressalta a importância da inovação das subsidiárias como forma de obtenção da vantagem competitiva da empresa multinacional (Meyer, Li \& Schotter, 2020). O desenvolvimento de inovações locais da subsidiária é um dos caminhos para o desenvolvimento de inovações de subsidiárias que possam ter alcance global (Gupta \& Govindarajan, 2001; Nohria \& Ghoshal, 1997). A inovação local é decorrência de ações internas da subsidiária, como, também, das condições de fatores do ambiente competitivo local (Porter, 1990; Birkinshaw, Hood \& Jonsson, 1998) e das redes locais (Andersson, Forsgren \& Holm, 2002). $O$ presente artigo enfoca fatores do ambiente externo que é uma das temáticas largamente estudadas no nível macro, ou seja, nacional.

No entanto, é reconhecido que o foco em fatores do macro ambiente do país ignora efeitos de fatores entre níveis subnacionais. As características locais subnacionais são consideradas relevantes, em especial, quando se trata de um país geograficamente amplo, onde se têm maiores disparidades (Falaster, Ferreira, Serra \& Storopoli, 2017; Bi, Ren \& Bao, 2020), tal como o Brasil. Esse olhar para as peculiaridades subnacionais pode indicar variações que uma análise em nível nacional pode desconsiderar por não levar em conta a heterogeneidade institucional subnacional (Hutzschenreuter, Matt \& Kleindienst, 2020; Li \& Sun, 2017; Yang, 2018; Meyer \& Nguyen, 2005).

Diante deste contexto, o presente artigo questiona quais são os efeitos dos fatores do ambiente subnacional brasileiro na criação de inovação local em subsidiárias estrangeiras? O estudo realizado em treze Estados brasileiros objetiva examinar os efeitos dos fatores do ambiente subnacional brasileiro - a eficiência do ambiente competitivo subnacional e o embeddedness relacional em cada localidade subnacional - na criação de inovação local em subsidiárias estrangeiras. As análises são realizadas por meio de uma abordagem quantitativa. Foram usados dados primários decorrentes da aplicação de uma survey quanto à percepção dos respondentes acerca dos fatores do macro ambiente. Assim como foram usados dados secundários de domínio público do World Bank Enterprise Surveys (WBES) para avaliar o efeito dos fatores institucionais subnacionais no nível da entidade federativa estadual.
A análise de fatores em nível subnacional oferece uma integração mais matizada das relações da subsidiária com o contexto local e aponta uma arena fértil tanto para os estudos de Negócios Internacionais (International Business - IB) quanto para a Geografia Econômica (GE) (Beugelsdijk \& Mudambi, 2013; Beleska-Spasova, Loykulnanta \& Nguyen, 2016; Meyer \& Nguyen, 2005; Yang, 2018; Hutzschenreuter et al., 2020). Ademais, Hutzschenreuter et al. (2020) conclamam o efeito dos fatores subnacionais na inovação das subsidiárias, tal como os estudos conceituais de Falaster e Ferreira (2020) e de Sun, Qu e Liao (2018), estes últimos, que examinam como as instituições subnacionais chinesas podem impactar a definição de incentivos ao investimento em pesquisa e desenvolvimento (P\&D). Nesse sentido, este estudo procura complementar o debate sobre o efeito do ambiente subnacional na inovação local das subsidiárias, tendo como contexto, o Brasil.

\section{FUNDAMENTAÇÃO TEÓRICA}

\subsection{Localização e inovação local}

Um dos fatores determinantes da inovação local das subsidiárias é o ambiente externo local. Meyer et al. (2020) mostram que o desenvolvimento de inovações é um dos principais tópicos de análise nos estudos de gestão de uma subsidiária. A inovação está associada ao papel da subsidiária na multinacional, que muda de acordo com os fatores dos ambientes locais da localização (Oliveira Jr., Boehe \& Borini, 2009). Isto é, o local onde a subsidiária está inserida é importante para o desenvolvimento de capacidades das subsidiárias (Meyer, Mudambi \& Narula, 2011; Andersson, Dellestrand \& Pedersen, 2014; Yang, 2018), ou seja, o desenvolvimento de inovação local na subsidiária (Birkinshaw et al., 1998).

O contexto local é composto por fatores do ambiente competitivo e das instituições. Esses fatores locais impactam nas escolhas de localização das subsidiárias (Chan, Makino \& Isobe, 2010; Ma, Tong \& Fitza, 2013; Li \& Sun, 2017; Kim \& Kim, 2018; Yang, 2018). Quanto melhor o ambiente local, maior a atração de subsidiárias, pois a região é mais propícia para a criação de inovação local (Rugman \& Oh, 2013; Porter, 1990; Andersson et al., 2002). Logo, a inovação local da subsidiária depende do quanto a empresa desfruta dos recursos do seu ambiente local 
Criação de Inovação Local por Subsidiárias Estrangeiras a partir de Vantagens Específicas do Ambiente Subnacional

(Gnyawali \& Hatfield, 2007). A contrapartida é verdadeira, pois a inovação local é desenvolvida para atender especificamente às necessidades do mercado onde a subsidiária atua de forma a garantir a sua competitividade (Costa, Borini \& Amatucci, 2013; Rugman \& Verbeke, 2001).

Em geral, os estudos abordam o local a partir de uma ótica macro, ou seja, por meio dos fatores do ambiente competitivo e das instituições nacionais. Estudos que examinam empiricamente como as subsidiárias respondem às mudanças subnacional receberam a devida atenção na literatura (Li \& Sun, 2017; Yang, 2018; Peng \& Beamish, 2019), mas apresentam crescimento nos últimos anos (Hutzschenreuter et al., 2020; Lu, Song, \& Shan, 2018). Isso por que ficam cada vez mais evidentes as peculiaridades regionais de cada nação (Falaster \& Ferreira, 2020; Batschauer, Floriani \& Amal, 2020). Quanto maior territorialmente e mais diversificada institucionalmente a nação, é esperada uma maior variabilidade do ambiente subnacional (Meyer \& Nguyen, 2005). Logo, apesar de estudos já denotarem a influência do ambiente nacional brasileiro na inovação das subsidiárias (Costa et al., 2013), assim como, das redes de negócios (Isaac, Borini, Raziq \& Benito, 2019), quando se fala de um país territorialmente grande como o Brasil, é de se esperar que exista uma variação no ambiente subnacional (WBES, 2017). Uma perspectiva de nível nacional, ou seja, que considera a homogeneidade espacial de um país pode camuflar regiões de maior atratividade dentro do país (Hutzschenreuter et al., 2020) porque alguns de seus Estados são menos vulneráveis e mais atrativos para investimentos e desenvolvimento de inovações (WBES, 2017). Deste modo, no presente artigo é verificado o efeito do ambiente subnacional na inovação local das subsidiárias.

\subsection{Ambiente competitivo subnacional}

A dinâmica do ambiente competitivo forma a base para a atração de multinacionais estrangeiras (Andersson et al., 2014; Beleska-Spasova et al., 2016; Rugman, Verbeke \& Nguyen, 2011). O ambiente competitivo é constituído por uma força agregada manifestada pelo grau de competição, rivalidade, pressão dos clientes e qualidade dos fornecedores (Porter, 1990), que tem o poder de impulsionar a inovação das subsidiárias (Birkinshaw \& Hood, 1998; Frost, Birkinshaw \& Ensign, 2002). Trata-se de fatores que dependem da trajetória do desenvolvimento local (Holm, Malmberg \& Sölvell, 2003), logo, de mudança evolutiva. De tal maneira determinam, também, a evolução das capacidades das firmas locais, tal como das subsidiárias, manifestada por meio de inovações em adaptação e criação de novos de produtos e processos (Andersson et al., 2014).

A inovação local das subsidiárias é impactada pelo ambiente competitivo, pois as interações entre uma subsidiária e outros atores locais potencializam as pressões do ambiente e os processos de aprendizado e adaptação (Porter, 1990; Andersson, Forsgren \& Holm, 2001; Andersson et al., 2002). Assim, a interação dinâmica no ambiente competitivo pode explicar em partes o sucesso e o fracasso das empresas instaladas nas economias emergentes (Chen \& Miller, 2012; Li \& Liu, 2014). Neste sentido, observa-se para o Brasil, dentre alguns dos indicadores da sofisticação do mercado, que o índice relativo ao comércio, competição e tamanho de mercado indica a existência de uma dinâmica forte, dado que o país ocupa a 32 a posição em comparação com cento e vinte e sete países no ranking global de inovação (Global Innovation Index - GII, 2017).

No entanto, a análise do ambiente competitivo pode ser mais bem entendida se ponderada pelas peculiaridades das instituições subnacionais, uma vez que pode explicar melhor como as empresas conseguem aproveitar os recursos à sua disposição para alcançar a inovação (Meyer \& Nguyen, 2005; Meyer et al., 2011; Goerzen, Asmussen \& Nielsen, 2013). As empresas optam por localizarem-se onde as instituições possibilitam um ambiente de negócios mais propício ao seu tipo de operação comercial e, em particular, onde as barreiras institucionais inibem menos o acesso aos recursos locais (Meyer \& Nguyen, 2005; Lu, Saka-Helmhout \& Piekkari, 2019; Hutzschenreuter et al., 2020). Assim, o nível de desenvolvimento institucional subnacional reflete a extensão em que as instituições políticas e econômicas em uma região subnacional favorecem ou não as empresas estrangeiras (Li \& Sun, 2017).

No nível subnacional, um elemento-chave das instituições relacionada à dinâmica do ambiente competitivo são as práticas concorrenciais, que consistem num dos obstáculos existentes no ambiente de negócios identificados pelo WBES (2009): a informalidade concorrencial. Trata-se da economia informal que abrange uma grande variedade de segmentos (Organização Internacional 
do Trabalho - OIT, 2018). É importante notar que no Brasil, segundo dados do Instituto Brasileiro de Geografia e Estatística (IBGE, 2018), em média nessa década aproximadamente $40 \%$ da população ocupada estava em trabalho informal. Ademais existe uma heterogeneidade da informalidade conforme as diferentes regiões do país (Souza, Trovão, Silva \& Melo, 2019). Por exemplo, Souza et al. (2019) mostram que em 2005 a taxa de informalidade aproximadamente na região Nordeste era de $71 \%$, na Norte de 68\%, na Centro-Oeste de 52\%, na Sul de 46\% e na Sudeste de 40\%. Após dez anos, em 2015 a informalidade caiu, mas as disparidades continuam: na região Nordeste era de $56 \%$, na Norte de $56 \%$, na Centro-Oeste de $34 \%$, na Sul de $25 \%$ e na Sudeste de $28 \%$.

Portanto a informalidade é um fator subnacional que tem efeito na dinâmica competitiva e, consequentemente, na estratégia de inovação das subsidiárias. A informalidade faz com que a concorrência tenha disparidade em termos da estrutura de custos. Ademais, a informalidade atua tanto a jusante, quanto a montante. As condições dos fornecedores da indústria são afetadas pela informalidade, assim como a demanda é impactada pela oferta de produtos oriundos da economia informal. Isso quer dizer que quanto menor a informalidade na região subnacional, maior a eficiência institucional e maior a probabilidade de impactar positivamente na competitividade da subsidiária (Li \& Sun, 2017). Logo,

Hipótese 1: A inovação local das subsidiárias estrangeiras está associada a maior eficiência do ambiente competitivo subnacional.

2.3. Relacionamento com parceiros de negócios no ambiente subnacional

De uma perspectiva local, as subsidiárias interagem externamente com outras organizações por meio de redes para atrair recursos (Meyer et al., 2020) para o desenvolvimento de competências (Andersson et al., 2002; Meyer et al., 2020) geralmente oriundas de inovações locais (Isaac et al., 2019). A teoria das redes de negócios reconhece que, em grande parte, os recursos críticos relacionados à inovação estão ligados às relações específicas da subsidiária com seus clientes, fornecedores, distribuidores e outras contrapartes locais, ou seja, com o embeddedness externo local (Andersson \& Forsgren, 1996; Andersson et al., 2002; Andersson,
Forsgren \& Holm, 2007). Isso porque a inserção da subsidiária numa rede externa propicia novos conhecimentos que podem auxiliá-la como fonte estratégica para o desenvolvimento de inovações em produtos, em processos e em serviços (Andersson et al., 2002; Figueiredo, 2011).

O embeddedness externo pode ser visto como um recurso em si porque através dele a empresa obtém acesso a recursos e capacidades que estão fora da organização. A possibilidade de uma subsidiária identificar novas informações, assimilar e transformar em processos produtivos depende fortemente da proximidade das relações existentes com diferentes parceiros comerciais (Andersson et al., 2002; Holm, Holmström \& Sharma, 2005; Andersson, Björkman \& Forsgren, 2005). Assim, o embeddedness externo abrange a relação das subsidiárias com seus clientes, fornecedores, distribuidores ou parceiros. Quando esta relação é permeada por transações baseadas em relacionamentos de alta qualidade e muito duradouros, os parceiros adaptam seus comportamentos extensivamente entre si baseados na confiança e no compromisso mútuo (embeddedness relacional) (Andersson et al., 2002; Hallin, Holm \& Sharma, 2011). O embeddedness relacional constitui a base para a partilha de conhecimento (Hallin et al., 2011; Nell \& Andersson, 2012) e criação da inovação local (Andersson et al., 2002; Isaac et al., 2019).

Ma et al. (2013) argumentam que o efeito da região subnacional no desempenho da subsidiária estrangeira é derivado do embeddedness da subsidiária no contexto local da região subnacional. Os parceiros fornecem informações confiáveis e úteis sobre o mercado, sobre outros parceiros locais potenciais, que ajudam a reduzir a incerteza e os riscos de colaboração (Kim \& Kim, 2018) auxiliando o processo de inovação. Desse modo é importante que existam parceiros qualificados para a subsidiária. Isto depende da qualificação da mão obra nas regiões subnacionais.

A qualificação da mão de obra de uma nação é a base para a produtividade das suas instituições. De Souza Barbosa e Lemos (2019) mostram que a escolaridade tem relação com a produtividade, porém com significativa variação dentro do Brasil. Os dados revelam que a menor qualificação educacional (menos anos de estudos) estão associados a menor produtividade, mostrando que a região Nordeste 
apresenta a menor produtividade e menor escolaridade, quando medida pelo porcentual da população com mais de 15 anos que completou apenas o ensino fundamental. Isso implica que os parceiros em diferente Estados da federação podem ter mais dificuldade para encontrar mão de obra qualificada e ter mais produtividade. A menor produtividade pode acarretar em menor disponibilidade para uma relação de troca de conhecimento entre parceiros e subsidiária (Andersson et al., 2002), o que pode implicar em menos predisposição para a inovação local. Logo,

Hipótese 2: A inovação local das subsidiárias estrangeiras está associada ao embeddedness relacional em cada localidade subnacional.

\section{METODOLOGIA}

\subsection{Coleta dos dados e amostra}

Os dados primários foram coletados por meio de uma survey enviada por e-mail para representantes com cargos de tomada de decisão das mil maiores multinacionais estrangeiras que operam no Brasil. Essa coleta foi realizada no primeiro semestre de 2009. Inicialmente foram consideradas 152 respostas para as variáveis sobre a atuação da subsidiária no Brasil, sendo a maior parte advinda América do Norte (30\%) e Europa (56\%), que atuam nos setores de agronegócios (3\%), indústria (76\%) e serviços (21\%), considerando as respostas completas e válidas.

Em posse dos dados foi verificado que as empresas respondentes atuam em treze Estados do Brasil, sendo que apenas 54 (35\%) atuam com operações em mais de um Estado. Quando a empresa atua em mais de um Estado, a proxy do ambiente subnacional é a média das notas obtidas nos Estados. Para os dados institucionais de cada Estado foram usados dados secundários, mais especificamente os dados do WBES. A base de dados do WBES (2009) fornece as respostas dadas por empresários e os principais gerentes em mil oitocentos e duas empresas que foram convidados a escolher o maior obstáculo para a realização de seus negócios. Esse conjunto de dados secundários permite distinguir os diferentes Estados do Brasil, incluindo os treze entes federativos em que as multinacionais da amostra atuam: Amazonas, Bahia, Ceará, Distrito Federal, Goiás, Mato Grosso, Minas Gerais, Paraná,
Pernambuco, Rio de Janeiro, Rio Grande do Sul, Santa Catarina e São Paulo. Importante salientar que para essa pesquisa foi usada a base de dados primários de 2009, pois os dados secundários relacionados aos obstáculos do ambiente de negócios subnacional do WBES datam, também, de 2009. Inclusive este foi o último período de coleta feito para o Brasil do WBES até o momento.

\subsection{Variáveis}

Todas as variáveis da survey estão medidas numa escala de Likert de cinco pontos, tendo-se num extremo o valor 1 , que indica discordo totalmente e, no outro extremo o valor 5 , concordo totalmente. Devido à impossibilidade de uma medida direta, os construtos latentes são representados por mais de uma variável latente, ou seja, por variáveis representadas e medidas por variáveis observáveis (Hair et al., 2009).

As variáveis do WBES também estão medidas numa escala de Likert de cinco pontos, na qual se tem num extremo o valor 0 , que indica que não há obstáculos e, no outro extremo o valor 4, que indica a existência de obstáculos muito severos. Em decorrência da diferença de escalas entre a survey e os dados do WBES, foi feito um ajuste de escalas com recodificação para que as respostas dadas ao WBES se adequassem à medição das respostas da survey. As escalas também foram invertidas para medir não o obstáculo do fator institucional, mas sim o quanto o fator é favorável para determinado Estado, de forma a combinar com a escala em sinalização positiva do ambiente nacional e de embeddedness.

\subsection{Variável dependente}

A variável dependente avalia a inovação local em subsidiárias de empresas multinacionais estrangeiras que operam em treze Estados do Brasil. O construto da inovação local baseia-se, em particular, na adaptação de Birkinshaw et al. (1998), para analisar se a subsidiária desenvolve inovações locais, partindo da premissa de que a autonomia para inovar localmente foi concedida pela matriz. A medição foi feita considerando que a inovação local das subsidiárias é dependente de uma trajetória evolutiva da adaptação para a criação de produtos e processos (Tarraço, Bernardes, Borini \& Rossetto, 2019). Foram considerados três itens da survey, perguntando se a empresa: (1) altera o design dos 
produtos / serviços oferecidos; (2) cria novos produtos / serviços; (3) altera os processos de produção. O Alfa de Cronbach, que mede a confiabilidade da consistência interna do constructo (Hair et al., 2009) é de 0,792.

\subsection{Variáveis independentes}

As variáveis independentes oriundas da survey são o embeddedness externo o ambiente competitivo.

O embeddedness externo refere-se aos relacionamentos e à confiança que a subsidiária desenvolve com seus clientes, fornecedores, distribuidores e parceiros. Esse constructo apoia-se em Andersson et al. (2002). A medição foi feita com seis itens da survey que perguntam: (1) o relacionamento com nossos principais parceiros é caracterizado por ser uma relação de longa duração; (2) o relacionamento com nossos principais parceiros é caracterizado por um alto nível de confiança mútua; (3) nossos principais parceiros nunca se comprometem a fazer coisas sem que venham a cumprir o prometido; (4) nossos principais parceiros normalmente compartilham conosco importantes informações sobre o mercado e vice e versa; (5) nós confiamos nos nossos parceiros no sentido de que eles irão entregar conforme o prometido e dentro do prazo determinado e (6) nós confiamos nos nossos parceiros no sentido de que eles irão entregar dentro do padrão de qualidade prometido. O Alfa de Cronbach é de 0,777.

O ambiente competitivo baseia-se em Porter (1990) e Birkinshaw et al. (1998). A medição foi feita com quatro itens da survey, perguntando a importância dos seguintes fatores para o desempenho da subsidiária: (1) tamanho da demanda de mercado; (2) exigência dos consumidores; (3) intensidade da competição e (4) velocidade na inovação de competidores. O Alfa de Cronbach é de 0,664.

Para calcular as variáveis do ambiente de negócios subnacional, utilizaram-se os dados coletados no WBES (2009) que indicam a existência dos obstáculos, selecionando-se para essa pesquisa apenas dois obstáculos para ponderar o efeito do ambiente competitivo e do embeddedness externo respondidos pelas subsidiárias. Para ponderar o efeito do ambiente competitivo escolheu-se o obstáculo da informalidade. Na survey do WBES a pergunta questiona até que ponto as práticas dos concorrentes no setor Informal são um obstáculo. A ponderação foi feita da seguinte forma: a nota média dos Estados em que a subsidiária atua de forma produtiva foi multiplicada pela nota média respondida para o ambiente competitivo. Por sua vez, para ponderar o efeito do embeddedness externo utilizou-se o obstáculo da inadequação da formação educacional da mão de obra. Na survey do WBES a pergunta questiona até que ponto uma força de trabalho com educação inadequada é um obstáculo. Da mesma forma, a ponderação foi feita com a nota média dos Estados em que a subsidiária atua multiplicada pela nota média respondida para o embeddedness externo.

\subsection{Variáveis de controle}

As variáveis de controle são a idade, o tamanho e o modo de entrada.

A idade considera o ano de abertura da subsidiária no respectivo Estado do Brasil e é medida em termos de número de anos, considerando o período entre 2000 e 2009 (Ano 00).

O tamanho da subsidiária no Estado onde ela opera no Brasil reflete a quantidade de funcionários que a empresa tem. Considerou-se para o controle, subsidiárias com menos que 100 funcionários (t100) porque subsidiárias menores provavelmente têm maior flexibilidade para inovar devido à menor burocracia interna e, também, porque suas inovações tendem a ser muito específicas para a demanda do mercado local (Costa et al., 2013).

O modo de entrada nos Estados dentro do mercado brasileiro pondera se a subsidiária optou por uma aquisição ou greenfield, uma vez que a literatura mostra que empresas adquiridas possuem mais autonomia do que empresas greenfield (Raziq, Borini, Perry \& Battisti, 2013) e, portanto, têm maior capacidade de inovar.

Para todas as variáveis de controle utilizou-se uma variável dummy atribuindo-se o valor 0 , caso a característica não esteja presente e 1, caso a característica esteja presente (Marôco, 2014; Fávero \& Belfiore, 2017). 


\section{RESULTADOS}

Os dados são analisados por meio da regressão linear múltipla. As respostas da survey feita para as subsidiárias são baseadas em percepção, da mesma forma que as do WBES. Entretanto, ao ponderar as respostas da survey pelas respostas do WBES, diminui-se o efeito da common method bias. Além disso, o teste de Harman para um único fator, mostra que a variância explicada é de $22 \%$, ou seja, bem abaixo dos 50\%. Para evitar o viés dos não respondentes a amostra foi dividida em duas partes dos $50 \%$ primeiros frente aos demais. A comparação das médias dos constructos usados no modelo não apresenta diferença significativa. Por fim, para aumentar a acurácia do modelo, além da modelagem com cento e cinquenta e dois respondentes (Modelo 1), foi realizada a rotação de um modelo com cento e trinta e sete observações (Modelo 2) oriundas das exclusões de casos com mais de dois desvio padrão dos resíduos.

A Tabela 1 mostra a média, o desvio padrão e as correlações (não padronizados) dos constructos pesquisados. A princípio, verifica-se, ainda que fraca, a correlação do ambiente competitivo subnacional com a inovação local da subsidiária.

Tabela 1: Descritivas e Correlações

\begin{tabular}{r|l|c|c|c|c|c}
\hline & & Média & Desvio Padrão & 1 & 2 & 3 \\
\hline 1 & Ambiente competitivo subnacional & 8,79 & 1,46 & 1 & & \\
\hline 2 & Embeddedness externo subnacional & 5,24 & 0,74 & $0,194^{*}$ & 1 & \\
\hline 3 & Inovação Local & 3,47 & 0,96 & $0,165^{*}$ & $-0,001$ & 1 \\
\hline & $* p<0,05$ & & & & & \\
\hline
\end{tabular}

Fonte: Elaborado pelos autores (2020).

A Tabela 2 mostra os testes para o comportamento das variáveis de controle (ano, modo de entrada e tamanho) e das variáveis independentes, e a relação destas com a variável dependente (inovação local) no Modelo 1 ( $n=152)$ e no Modelo 2 ( $n=137)$. Os modelos apresentam VIF menor que cinco, ou seja, são ausentes de multicolinearidade.

Tabela 2. Modelos de Regressão

\begin{tabular}{l|c|c}
\hline & Modelo 1 & Modelo 2 \\
\hline Ano & 0,037 & 0,006 \\
\hline Aquisição & $0,198 *$ & $0,249 * *$ \\
\hline Tamanho & $-0,084$ & $-0,019$ \\
\hline Ambiente competitivo subnacional & $0,202 *$ & $0,376 * *$ \\
\hline Embeddedness externo subnacional & $-0,024$ & $-0,099$ \\
\hline \multirow{2}{*}{$\mathrm{R}^{2}$ Ajustado } & 0,045 & 0,14 \\
\hline \hline
\end{tabular}

Variável dependente: Inovação local.

Fonte: Elaborado pelos autores (2020).

Os resultados mostram que existe uma associação positiva e significante entre o ambiente competitivo subnacional e inovação local das subsidiárias. Esse resultado confirma a hipótese 1 . Por sua vez, tanto no Modelo 1, como no Modelo 2 não é observada uma associação significativa entre 0 embeddedness externo subnacional e a inovação local. Deste modo a hipótese 2 não é suportada. Ainda, os dois modelos mostram que as empresas que entram por meio de aquisições estão mais associadas à inovação local, o 
que confirma o pressuposto que subsidiárias constituídas por aquisições possuem mais autonomia para a inovação local (Raziq et al. 2013).

\section{DISCUSSÃO}

Os resultados confirmam que o ambiente competitivo subnacional $(\mathrm{H} 1)$ exerce um efeito significativo para as subsidiárias estrangeiras criarem inovações locais, o que está de acordo com a literatura e destaca a importância de se estudar o contexto subnacional (Chan et. al, 2010; Beugelsdijk \& Mudambi, 2013; Korez-Vide, Voller \& Bobek, 2014; Beleska-Spasova et al., 2016; Goerzen et al., 2013; Yang, 2018; Hutzschenreuter et al., 2020). Esse resultado ressalta a escolha da localização (Ma et al., 2013; Falaster et al., 2017; Li \& Sun, 2017; Yang, 2018; Kim \& Kim, 2018), em especial, quanto à dinâmica competitiva como um fator importante para o propósito estratégico de inovação das subsidiárias estrangeiras, ao menos, em território brasileiro. Quanto mais dinâmica concorrência, demanda e indústria de fatores, e menor a interferência da economia informal, mais as subsidiárias tendem a voltar seus objetivos estratégicos para a inovação local. Este resultado está alinhado com a importância do ambiente subnacional, mostrando que a inovação tende a proliferar onde as instituições são mais propícias às operações de negócios e onde as barreiras institucionais inibem menos o acesso aos recursos locais (Meyer \& Nguyen, 2005; Kim \& Kim, 2018).

Por sua vez, os resultados não apresentam a associação do embeddedness subnacional com a inovação local das subsidiárias estrangeiras $(\mathrm{H} 2)$. Uma das explicações é que a mobilidade da mão de obra pode ajudar as empresas de diferentes Estados a completar seus quadros qualificados, uma vez que a literatura (Isaac et. al, 2019) mostra a associação do embeddedness no nível nacional. Outra razão é que, como a análise é baseada em dados de treze Estados brasileiros, é possível afirmar que nesses Estados os obstáculos não influenciam a criação de inovação local por subsidiárias estrangeiras, mas variações institucionais diversas podem ser encontradas em outros Estados da federação (Chan et al., 2010; Meyer \& Nguyen, 2005; Goerzen et al., 2013; WBES, 2017).

Além da análise estrita das hipóteses, os resultados são importantes porque demonstram que em um mercado emergente, o ambiente competitivo subnacional tem efeito sob a inovação local. Isso implica que a unidade federativa versus a autonomia estadual deve ser muito bem pesada e balanceada para dirimir distorções maiores daquelas que uma economia emergente como o Brasil já apresenta pela sua trajetória de desenvolvimento institucional.

Em termos de contribuição acadêmica, destaca-se que, embora existam indícios do impacto do ambiente institucional na estratégia e inovação, este estudo no âmbito brasileiro avança em dois pontos. Primeiro, os estudos analisam mercados desenvolvidos (Santangelo et al., 2016) e mercados emergentes (Chan et al., 2010; Meyer \& Nguyen, 2005), porém esses últimos de forma pontual e numa realidade asiática (China e Vietnã), que apresenta uma considerável diferença do mercado latino americano e brasileiro, destacando-se as peculiaridades do ambiente subnacional brasileiro. Por exemplo, o impacto das instituições econômicas subnacionais no Brasil (mercado emergente) tem suas peculiaridades frente os mercados desenvolvidos, ou mesmo, em relação à China (Khanna \& Palepu, 2011; Sull \& Escobari, 2004; Sull \& Wang, 2005). Segundo, contribui para os estudos de geografia econômica (McCann \& Mudambi, 2005) reforçando que cada ambiente subnacional tem suas peculiaridades, como foi mostrado na natureza das questões subnacionais no Brasil.

Em termos gerenciais, o estudo chama a atenção para a importância do conhecimento da dinâmica competitiva subnacional em especial quanto à participação da economia informal. Nesse sentido estende as implicações para políticas públicas. Para os governantes os resultados demonstram a importância do fortalecimento do ambiente institucional do sistema nacional de inovação, evidenciando a necessidade de ações públicas que governos estaduais e municipais devem pensar para figurar seu ambiente subnacional como polo de atratividade para o investimento estrangeiro.

\section{CONCLUSÃO}

Os ambientes subnacionais estudados atuam como um polo de atração das subsidiárias que desejam realizar localmente atividades específicas de criação de inovação local, principalmente em Estados onde países anfitriões como o Brasil têm vantagens específicas no ambiente competitivo subnacional. Os 
achados trazem contribuições à pesquisa de negócios internacionais e de geografia econômica para a inovação local de subsidiárias. Retratam a importância do estudo sobre o ambiente de negócios subnacional, representado nesta pesquisa pelo estudo de treze Estados do Brasil. As descobertas são relevantes para o estudo de subsidiárias estrangeiras que operam em economias emergentes, porque utilizam a subsidiária como unidade de análise e também o ambiente subnacional, contribuindo para o preenchimento da lacuna de pesquisa levantada.

A pesquisa tem limitações. A primeira diz respeito ao período da coleta dos dados. A base de dados primários é datada de 2009 porque a última coleta de dados secundários realizada pelo WBES sobre os obstáculos do ambiente subnacional brasileiro foi feita em 2009, o que inviabiliza a realização de uma nova survey para atualizar os dados da pesquisa, considerando a inexistência de dados atuais. A segunda limitação se relaciona ao recorte transversal de 2009 que pode não acompanhar algumas transformações politicas e econômicas dos anos subsequentes, que podem ter impactado não apenas os obstáculos pesquisados, mas alterado as condições com as quais os representantes das subsidiárias responderam à survey.

Como sugestão de estudos futuros, recomenda-se a replicação do método para avaliar se os resultados encontrados reaparecem nos tempos atuais. Para isso, como o WBES não tem novos dados disponíveis, sugere-se que os pesquisadores incluam em suas surveys as mesmas questões efetivadas para a composição dos constructos apresentados nesta pesquisa e adicionem as questões para medir a percepção dos gestores das subsidiárias sobre quão severos os obstáculos presentes no ambiente de negócios subnacional se apresentam e qual a sua influência na capacidade das empresas de criar inovações locais, baseando-se nos indicadores de medição utilizados pelo WBES, cujo questionário está disponível no site do próprio órgão.

\section{AGRADECIMENTOS}

Este estudo foi financiado em parte pela Coordenação de Aperfeiçoamento de Pessoal de Nível Superior - Brasil (CAPES) - Código de financiamento 001.

\section{REFERÊNCIAS}

Andersson, U., \& Forsgren, M. (1996). Subsidiary embeddedness and control in the multinational corporation. International Business Review, 5(5), 487508.

Andersson, U., Björkman, I., \& Forsgren, M. (2005). Managing subsidiary knowledge creation: The effect of control mechanisms on subsidiary local embeddedness. International Business Review, 14(5), 521-538.

Andersson, U., Dellestrand, H., \& Pedersen, T. (2014). The contribution of local environments to competence creation in multinational enterprises. Long Range Planning, 47(1-2), 87-99.

Andersson, U., Forsgren, M., \& Holm, U. (2001). Subsidiary embeddedness and competence development in MNEs a multi-level analysis. Organization Studies, 22(6), 1013-1034.

Andersson, U., Forsgren, M., \& Holm, U. (2002). The strategic impact of external networks: subsidiary performance and competence development in the multinational corporation. Strategic Management Journal, 23(11), 979-996.

Andersson, U., Forsgren, M., \& Holm, U. (2007). Balancing subsidiary influence in the federative MNE: a business network view. Journal of International Business Studies, 38(5), 802-818.

Batschauer da Cruz, C.B., Eliete Floriani, D., \& Amal, M. (2020). The OLI Paradigm as a comprehensive model of FDI determinants: a sub-national approach. International Journal of Emerging Markets, Vol. aheadof-print No. ahead-of-print.

Beleska-Spasova, E., Loykulnanta, S., \& Nguyen, Q. T. (2016). Firm-specific, national and regional competitive advantages: The case of emerging market MNEsThailand. Asian Business \& Management, 15(4), 264291.

Beugelsdijk, S., \& Mudambi, R. (2013). MNEs as border-crossing multi-location enterprises: The role of discontinuities in geographic space. Journal of International Business Studies, 44(5), 413.

Bi, Y., Ren, Z., \& Bao, K. (2020). Does distance matter in foreign direct investment sub-national location choice? Evidence from China. Frontiers of Business Research in China, 14, 1-19. 
Birkinshaw, J., \& Hood, N. (1998). Multinational subsidiary evolution: Capability and charter change in foreign-owned subsidiary companies. Academy of Management Review, 23(4), 773-795.

Birkinshaw, J., Hood, N., \& Jonsson, S. (1998). Building firm-specific advantages in multinational corporations: the role of subsidiary initiative. Strategic Management Journal, 19(3), 221-242.

Chan, C. M., Makino, S., \& Isobe, T. (2010). Does subnational region matter? Foreign affiliate performance in the United States and China. Strategic Management Journal, 31(11), 1226-1243.

Chen, M. J., \& Miller, D. (2012). Competitive dynamics: Themes, trends, and a prospective research platform. The Academy of Management Annals, 6(1), 135-210.

Costa, S., Borini, F. M., \& Amatucci, M. (2013). Inovação global de subsidiárias estrangeiras localizadas em mercados emergentes. Revista de Administração Contemporânea, 17(4), 459-478.

de Sousa Barbosa, V., \& Lemos, J. D. J. S. (2019). Diferencial de capital humano induzem desigualdades nos diferencial de produtividade do trabalho entre os estados brasileiros. Desenvolvimento em Debate, 7 (2), 11-27.

Falaster, C. D., Ferreira, M. P., Serra, F. A. R., \& Storopoli, J. (2017). Institutional differences between regions within a country and ownership decisions in cross-border acquisitions. 3ES - Anpad, São Paulo: Anpad, 1-8.

Falaster, C., \& Portugal Ferreira, M. (2020). Institutional factors and subnational location choice for multinationals' R\&D subsidiaries. Innovation \& Management Review, Vol. ahead-of-print No. ahead-ofprint.

Fávero, L. P., \& Belfiore, P. (2017). Manual de análise de dados: estatística e modelagem multivariada com Excel $^{\circledR}$, SPSS $^{\circledR}$ e Stata $^{\circledR}$. Elsevier Brasil.

Figueiredo, P. N. (2011). The role of dual embeddedness in the innovative performance of MNE subsidiaries: evidence from Brazil. Journal of Management Studies, 48(2), 417-440.
Frost, T. S., Birkinshaw, J. M., \& Ensign, P. C. (2002). Centers of excellence in multinational corporations. Strategic Management Journal, 23(11), 997-1018.

Global Innovation Index. (2017). Report. Disponível em: https://www.globalinnovationindex.org/gii-2017report\#. Acesso em agosto, 2017.

Gnyawali, D. R., \& Hatfield, D. E. (2007). Foreign subsidiaries' learning from local environments: An empirical test. Management International Review, 47(1), 79-102.

Goerzen, A., Asmussen, C. G., \& Nielsen, B. B. (2013). Global cities and multinational enterprise location strategy. Journal of International Business Studies, 44(5), 427-450.

Hair, J. F., Black, W. C., Babin, B. J., Anderson, R. E., \& Tatham, R. L. (2009). Análise multivariada de dados. Bookman Editora.

Gupta, A. K., \& Govindarajan, V. (2001). Global dominance: transforming global presence into global competitive advantage. San Francisco: Jossey-Bass.

Hallin, C., Holm, U., \& Sharma, D. D. (2011). Embeddedness of innovation receivers in the multinational corporation: Effects on business performance. International Business Review, 20(3), 362373.

Holm, U., Holmström, C., \& Sharma, D. (2005). Competence development through business relationships or competitive environment?-subsidiary impact on MNE competitive advantage. MIR: Management International Review, 197-218.

Holm, U., Malmberg, A., \& Sölvell, Ö. (2003). Subsidiary impact on host-country economies-The case of foreign-owned subsidiaries attracting investment into Sweden. Journal of Economic Geography, 3(4), 389-408.

Hutzschenreuter, T., Matt, T., \& Kleindienst, I. (2020). Going subnational: A literature review and research agenda. Journal of World Business, 55(4), 101076.

Instituto Brasileiro de Geografia e Estatística. (2018). Síntese de indicadores sociais: uma análise das condições de vida da população brasileira (Estudos e pesquisas. Informação demográfica e socioeconômica, n. 39). Rio de Janeiro, RJ: Autor. Disponível em: 
https://biblioteca.ibge.gov.br/visualizacao/livros/liv101 629.pdf. Acesso em setembro, 2020.

Isaac, V. R., Borini, F. M., Raziq, M. M., \& Benito, G. R. (2019). From local to global innovation: The role of subsidiaries' external relational embeddedness in an emerging market. International Business Review, 28(4), 638-646.

Khanna, T., \& Palepu, K. G. (2011). Winning in emerging markets: Spotting and responding to institutional voids. World Financial Review, (May-June 2011), 18-20.

Kim, J., \& Kim, K. (2018). How does local partners network embeddedness affect international joint venture survival in different subnational contexts?. Asia Pacific Journal of Management, 35(4), 1055-1080.

Korez-Vide, R., Voller, P., \& Bobek, V. (2014). German and Austrian foreign direct investment in Brazilian regions: which are the location choice factors?. Journal of Management and Strategy, 5(4), 68-81.

Li, D. Y., \& Liu, J. (2014). Dynamic capabilities, environmental dynamism, and competitive advantage: Evidence from China. Journal of Business Research, 67(1), 2793-2799.

Li, X., \& Sun, L. (2017). How do sub-national institutional constraints impact foreign firm performance?. International Business Review, 26(3), 555-565.

Lu, J. W., Song, Y., \& Shan, M. (2018). Social trust in subnational regions and foreign subsidiary performance: Evidence from foreign investments in China. Journal of International Business Studies, 49(6), 761-773.

Lu, W., Saka-Helmhout, A., \& Piekkari, R. (2019). Adaptation of compensation practice in China: The role of sub-national institutions. Management and Organization Review, 15(2), 235-267.

Ma, X., Tong, T. W., \& Fitza, M. (2013). How much does subnational region matter to foreign subsidiary performance? Evidence from Fortune Global 500 Corporations' investment in China. Journal of International Business Studies, 44(1), 66-87.

Marôco, J. (2014). Análise Estatística com o SPSS Statistics. ReportNumber, Lda.
McCann, P., \& Mudambi, R. (2005). Analytical differences in the economics of geography: the case of the multinational firm. Environment and Planning $A$, 37(10), 1857-1876.

Meyer, K. E., \& Nguyen, H. V. (2005). Foreign investment strategies and sub-national institutions in emerging markets: Evidence from Vietnam. Journal of Management Studies, 42(1), 63-93.

Meyer, K. E., Li, C., \& Schotter, A. P. (2020). Managing the MNE subsidiary: Advancing a multi-level and dynamic research agenda. Journal of International Business Studies, 1-39.

Meyer, K. E., Mudambi, R., \& Narula, R. (2011). Multinational enterprises and local contexts: The opportunities and challenges of multiple embeddedness. Journal of Management Studies, 48(2), 235-252.

Nell, P. C., \& Andersson, U. (2012). The complexity of the business network context and its effect on subsidiary relational (over-) embeddedness. International Business Review, 21(6), 1087-1098.

Nohria, N., \& Ghoshal, S. (1997). The differentiated network: Organizations knowledge flows in multinational corporations. San Francisco: Jossey-Bass.

Organização Internacional do Trabalho. (2018). Futuro do Trabalho no Brasil: Perspectivas e Diálogos Tripartites. Disponível em: https://www.ilo.org/wcmsp5/groups/public/--americas/---ro-lima/---ilobrasilia/documents/publication/wcms 626908.pdf. Acesso em fevereiro, 2019.

Oliveira Jr, M. M., Boehe, D. M., \& Borini, F. M. (2009). Estratégia e inovação em corporações multinacionais: a transformação das subsidiárias brasileiras. São Paulo: Saraiva.

Peng, G. Z., \& Beamish, P. W. (2019). Subnational FDI legitimacy and foreign subsidiary survival. Journal of International Management, 25(3), 100662.

Porter, M. E. (1990). The competitive advantage of nations. Harvard business review, 68(2), 73-93.

Raziq, M. M., Borini, F. M., Perry, M., \& Battisti, M. (2013). Subsidiary characteristics and impact on subsidiary strategic and operational autonomy. Journal of Transnational Management, 18(3), 219-241. 
Rugman, A. M., \& Oh, C. H. (2013). Why the home region matters: location and regional multinationals. British Journal of Management, 24(4), 463-479.

Rugman, A. M., \& Verbeke, A. (2001). Subsidiaryspecific advantages in multinational enterprises. Strategic Management Journal, 22(3), 237-250.

Rugman, A. M., Verbeke, A., \& Nguyen, Q. T. (2011). Fifty years of international business theory and beyond. Management International Review, 51(6), 755-786.

Santangelo, G. D., Meyer, K. E., \& Jindra, B. (2016). MNE subsidiaries' outsourcing and insourcing of R\&D: The role of local institutions. Global Strategy Journal, 6(4), 247-268.

Souza, D. M, Trovão, C. J. B. M, Silva, M. R, \& Melo, J. W. F. (2019). Informalidade no Brasil: aspectos socioeconômicos dos trabalhadores informais sob a ótica regional. Interface - Revista do Centro de Ciências Sociais Aplicadas, 16 (1), 142-165.

Sull, D. N., \& Escobari, M. (2005). Success against the odds: what Brazilian champions teach us about thriving in unpredictable markets. Rio de Janeiro and Cambridge, Massachusetts: Editora Campus.

Sull, D. N., \& Wang, Y. (2005). Made in China: What Western managers can learn from trailblazing Chinese entrepreneurs. Harvard Business Press.
Sun, P., Qu, Z., \& Liao, Z. (2018). How and when do subnational institutions matter for R\&D investment? Evidence from the Chinese pharmaceutical sector. IEEE Transactions on Engineering Management, 65(3), 379391.

Tarraço, E.L., Bernardes, R.C., Borini, F.M., \& Rossetto, D.E. (2019). Innovation capabilities for global R\&D projects in subsidiaries. European Journal of Innovation Management, 22 (4), 639-659.

World Bank Group Enterprise Surveys. (2009). Enterprise Surveys what businesses experience. Disponível em: https://www.enterprisesurveys.org/data/exploreecono mies/2009/brazil. Acesso em junho, 2017.

World Bank Group Enterprise Surveys. (2009). Ranking of the top business environment obstacle for firms. Disponível em: https://www.enterprisesurveys.org/en/data/exploreec onomies/2009/brazil\#2. Acesso em junho, 2017.

World Bank Group Enterprise Surveys. (2017). Indicator Descriptions. Disponível em: https://www.enterprisesurveys.org/data/exploreecono mies/2009/ /media/GIAWB/EnterpriseSurveys/Docum ents/Misc/Indicator-Descriptions.pdf. Acesso em setembro, 2017.

Yang, J. (2018). Subnational institutions and location choice of emerging market firms. Journal of International Management, 24(4), 317-332.

\section{SOBRE OS AUTORES:}

Cristina Doritta Rodrigues - Escola Superior de Propaganda e Marketing - ESPM, São Paulo, (Brasil). E-mail: crisdoritta@gmail.com Orcid id: http://orcid.org/0000-0002-6999-8855

Felipe Mendes Borini - Universidade de São Paulo - USP, São Paulo, (Brasil). E-mail: fborini@usp.br Orcid id: http://orcid.org/0000-0003-1389-136X

Muhammad Mustafa Raziq - National University of Sciences and Technology, Islamabad (Pakistan). E-mail: mustafa.raziq@nbs.nust.edu.pk Orcid id: http://orcid.org/0000-0002-7793-1597

Manuel Portugal Ferreira - Universidade Federal de Lavras - UFLA, Minas Gerias (Brasil). E-mail: manuel.portugal.ferreira@gmail.com Orcid id: http://orcid.org/0000-0002-4642-4605 


\title{
CREATION OF LOCAL INNOVATION BY FOREIGN SUBSIDIARIES FROM SPECIFIC ADVANTAGES OF THE SUBNATIONAL ENVIRONMENT
}

\author{
Cristina Doritta Rodrigues ${ }^{\Omega}$, Felipe Mendes Borini ${ }^{\ddagger}$, Muhammad Mustafa Raziq ${ }^{\ddagger}$ e \\ Manuel Portugal Ferreira ${ }^{€}$ \\ $\Omega$ Escola Superior de Propaganda e Marketing - ESPM, São Paulo, (Brasil) \\ £ Universidade de São Paulo - USP, São Paulo, (Brasil) \\ ${ }^{¥}$ National University of Sciences and Technology, Islamabad (Pakistan) \\ €Universidade Federal de Lavras - UFLA, Minas Gerais (Brasil)
}

\begin{tabular}{l}
\hline ARTICLE DETAILS \\
\hline Article history: \\
Received: 30 March 2020 \\
Accepted: 17 October 2020 \\
Available online January: 01 th 2021 \\
Double Blind Review System \\
Scientific Editor \\
Ilan Avrichir
\end{tabular}

\section{Keywords}

Foreign subsidiaries

Subnational environment

Local innovation

Multiple linear regression

\begin{abstract}
Purpose - The study aims to examine the effects of factors in the Brazilian subnational environment - the efficiency of the subnational competitive environment and the relational embeddedness in each subnational location in the creation of local innovation in foreign subsidiaries.
\end{abstract}

Method - The approach is quantitative and the research is based on primary data collected through a survey of 152 foreign subsidiaries located in thirteen states in Brazil and secondary data from World Bank Enterprise Surveys. The data are analyzed by multiple linear regression.

Main results - The results show that there is a positive and significant association between the subnational competitive environment and the local innovation of the subsidiaries. However, there is no significant association between subnational external embeddedness and local innovation. In addition, the results show that companies that enter through acquisitions are more associated with local innovation.

Relevance/Originality - The study seeks to complement the debate on the effect of the subnational environment on local innovation in subsidiaries with Brazil as a context. Subnational level analysis considers subnational institutional heterogeneity and reveals variations that could be disregarded by national level analysis.

Theoretical/methodological contributions: Although there is evidence of the impact of the institutional environment on strategy and innovation, this study at the Brazilian level advances on two points. First, the existing studies analyze developed markets and emerging markets, but the latter in a timely manner and in an Asian reality, which presents a considerable difference from the Latin American and Brazilian market, highlighting the peculiarities of the Brazilian subnational environment. Second, the research contributes to the studies of Economic Geography, reinforcing that each subnational environment has its particularities, as demonstrated in the nature of subnational issues in Brazil.

Social / management contributions: In managerial terms, the study draws attention to the importance of knowledge of the subnational competitive dynamics regarding the participation of the informal economy and extends implications for public policies. 


\title{
CREACIÓN DE INNOVACIÓN LOCAL POR SUBSIDIARIAS EXTRANJERAS A PARTIR DE VENTAJAS ESPECÍFICAS DEL ENTORNO SUBNACIONAL
}

\author{
Cristina Doritta Rodrigues ${ }^{\Omega}$, Felipe Mendes Borinif ${ }^{\ddagger}$, Muhammad Mustafa Raziq ${ }^{\ddagger}$ e \\ Manuel Portugal Ferreira ${ }^{€}$ \\ $\Omega$ Escola Superior de Propaganda e Marketing - ESPM, São Paulo, (Brasil) \\ £ Universidade de São Paulo - USP, São Paulo, (Brasil) \\ ${ }^{¥}$ National University of Sciences and Technology, Islamabad (Pakistan) \\ €Universidade Federal de Lavras - UFLA, Minas Gerais (Brasil)
}

\section{HISTORIA DEL ARTÍCULO}

\section{Historia del Artículo:}

Recibido: 30 de Marzo de 2020

Aceptado: 17 de Octubre de 2020

Disponible en línea: 01 de Enero 2021

Double Blind Review System

Editor Científico

Ilan Avrichir

\section{Palabras-clave:}

Filiales extranjeras

Ambiente subnacional

Innovación local

Regresión lineal múltiple

\section{RESUMEN}

Objetivo - El estudio tiene como objetivo examinar los efectos de los factores en el entorno subnacional brasileño - la eficiencia del entorno competitivo subnacional y el arraigo relacional en cada ubicación subnacional - en la creación de innovación local en las filiales extranjeras.

Método - El enfoque es cuantitativo y la investigación se basa en datos primarios recopilados a través de una encuesta de 152 subsidiarias extranjeras ubicadas en trece estados de Brasil y datos secundarios de Encuestas de Empresas del Banco Mundial. Los datos se analizan mediante regresión lineal múltiple.

\begin{abstract}
Resultados principales - Los resultados muestran que existe una asociación positiva y significativa entre el entorno competitivo subnacional y la innovación local de las filiales. Sin embargo, no existe una asociación significativa entre la integración externa subnacional y la innovación local. Además, los resultados muestran que las empresas que ingresan al ámbito subnacional mediante adquisiciones están más asociadas a la innovación local.
\end{abstract}

Relevancia/Originalidad: El estudio busca complementar el debate sobre el efecto del entorno subnacional en la innovación local en filiales con Brasil como contexto. El análisis a nivel subnacional considera la heterogeneidad institucional subnacional y revela variaciones que podrían ser ignoradas por el análisis a nivel nacional.

\begin{abstract}
Contribuciones teóricas/metodológicas - Si bien existen indicios del impacto del entorno institucional en la estrategia y la innovación, este estudio a nivel brasileño avanza en dos puntos. Primero, los estudios existentes analizan mercados desarrollados y mercados emergentes, pero estos últimos de manera oportuna y en una realidad asiática, que presenta una diferencia considerable con el mercado latinoamericano y brasileño, destacando las peculiaridades del entorno subnacional brasileño. En segundo lugar, contribuye a los estudios de Geografía Económica, reforzando que cada entorno subnacional tiene sus particularidades, como lo demuestra la naturaleza de los temas subnacionales en Brasil.

Contribuciones sociales / de gestión: En términos gerenciales, el estudio llama la atención sobre la importancia del conocimiento de la dinámica competitiva subnacional en torno a la participación de la economía informal y amplía las implicaciones para las políticas públicas.
\end{abstract}

\section{Como citar este artigo:}

Rodrigues, C., Borini, F., Raziq, M., \& Ferreira, M. (2021). Criação de Inovação Local por Subsidiárias Estrangeiras a partir de Vantagens Específicas do Ambiente Subnacional. Internext, 16(1), 75-88. doi: http://dx.doi.org/10.18568/internext.v16i1.600 